\title{
ANALYSIS OF A MODEL BIOLOGICAL SWITCH*
}

\author{
W. L. KATH $\dagger$ AND J. D. MURRAY $\ddagger$
}

\begin{abstract}
A model mechanism proposed by Murray [Phil. Trans. Roy. Soc. London B, 295 (1981), pp. 473-496] for generating wing patterns and eyespots on butterflies and moths is based on a morphogen $(S)$ activated biological switch for a gene product $(g)$. We analyse one of the resulting partial differential equation systems, namely

$$
S_{t}=D \Delta S-k S, \quad g_{t}=k_{1} S+\alpha g\left(g-k_{2}\right)\left(g_{c}-g\right),
$$

where $D, k, k_{1}, k_{2}, g_{c}>k_{2}$ and $\alpha$ are positive constants. We determine analytically the size of the spatial domain where $g \rightarrow g_{c}$ as $t \rightarrow \infty$ after an influx of $S$ at the origin. This gives the size of the eyespot in terms of the mechanism parameters. The analytical problem is a nontrivial singular perturbation expansion which we discuss in detail.
\end{abstract}

1. Model mechanism. The development of patterns and the pattern formation processes are of paramount importance in developmental biology. In particular the development of wing patterns in lepidoptera (butterflies and moths) is again of current interest (Nijhout [5]) since it presents a dramatic example of biological pattern formation amenable to quantitative controlled experiment (Nijhout [6], [7]) and mathematical modelling (Murray [4]).

Seminal work on the formation of wing eyespots has been done by Nijhout [6] who presents evidence for the centre of the eyespot as a source of some morphogen which diffuses in a radially symmetric way over the pupal wing. Murray [4] suggested that this morphogen activates a color-specific enzyme in a threshold manner and hence produces a pre-pattern for eyespot determination. The mechanism involves a biological switch. Preliminary results [4] from numerical studies gave encouraging results when compared with experiment. Quantitative behaviour of the pattern as a function of the model parameters is required and is the problem which this paper addresses.

The biological switch model for eyespot formation is the following. A source of morphogen of concentration $S^{*}\left(r^{*}, t^{*}\right)$ is released at $r^{*}=0$, the eyespot focus, which then diffuses across the two-dimensional wing surface with constant diffusion coefficient $D$ and degrades according to first order kinetics with rate $k$. The governing equation for $S^{*}$ is then

$$
S_{t^{*}}^{*}=D \Delta^{*} S^{*}-k S^{*} .
$$

As $S^{*}$ diffuses across the wing, the cells react to the local morphogen level and a gene is activated to produce a product of concentration $g^{*}$. The kinetics of the gene production exhibits a biochemical switch behaviour which can effect a permanent change in the product level, thus producing a spatial pattern in $g^{*}$. The specific model suggested by Murray [4] is

$$
\frac{d g^{*}}{d t^{*}}=k_{1} S^{*}+\frac{k_{2} g^{* 2}}{1+g^{* 2}}-k_{3} g^{*}=f\left(g^{*}\right)
$$

* Received by the editors December 20, 1983, and in final revised form February 15, 1985. This work was partially supported by the National Science Foundation under grant NCS-8205407 and by the U.S. Army Research Office under contract DAAG29-81-K-0107.

$\dagger$ Applied Mathematics 217-50 California Institute of Technology, Pasadena, California 91125. Present address, Department of Engineering Sciences and Applied Mathematics, The Technological Institute, Northwestern University, Evanston, Illinois 60201.

\$Centre for Mathematical Biology, Mathematical Institute, 24-29 St. Giles, Oxford, England OX1 3LB. 
The $k_{1} S^{*}$ represents activation by the morphogen, the $k_{2}$-term is a self-activation one with positive feedback while the $k_{3}$-term is linear degradation. For $k_{2}>2 k_{3}$ there are three possible steady states for $g^{*}$ : see Fig. 1 .

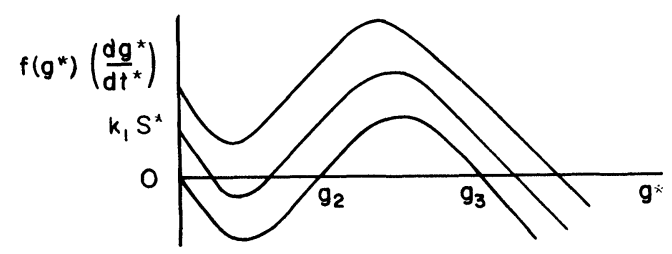
large.

FIG. 1. Schematic switch mechanism from $g^{*}=0$ to $g^{*}=g_{3}$ if the maximum of the $k_{1} S^{*}$-pulse is sufficiently

In the biological situation of interest a given amount of morphogen is released at $r^{*}=0$, at $t^{*}=0$, or over a period $t_{0}$, and it diffuses radially across the plane wing surface. Initially $g^{*}=0$. As $S^{*}$ diffuses, it activates the cell to produce $g^{*}$ which increases according to the last equation: see also Fig. 1. If $S_{\max }^{*}$ of the morphogen pulse is not sufficiently large, then $S^{*} \rightarrow 0$ and $g^{*} \rightarrow 0$ again as $t^{*} \rightarrow \infty$. On the other hand if at any position $r^{*}, S_{\max }^{*}$ is sufficiently large for a sufficient time then $g$ can increase enough so that it tends to the steady state $g^{*}=g_{3}$ and it will ultimately remain there after $S^{*}$ again tends to zero. The question of biological interest is: What is the size of the gene-activated domain as a function of the parameters and the amount and size of the morphogen pulse released at the focus?

In the rest of the paper we consider, in place of $f\left(g^{*}\right)$ in Fig. 1, a cubic in $g^{*}$, which is qualitatively similar. We do this for algebraic simplicity only: the analysis can be carried out for general $f\left(g^{*}\right)$. Specifically we take as the gene product activation switch

$$
\frac{d g^{*}}{d t^{*}}=k_{1} S^{*}+\alpha g^{*}\left(g^{*}-k_{2}^{*}\right)\left(g_{c}-g^{*}\right)
$$

where $k_{1}, k_{2}^{*}, g_{c}>k_{2}^{*}$ and $\alpha$ are positive constants. The equivalent of $g_{3}$ is now $g^{*}=g_{c}$.

In $\S 2$ we present some preliminary analysis in which we consider the gene activation kinetics to be at equilibrium, that is we consider the reaction time to be negligible compared with the morphogen reaction time scale. We obtain analytical results for the activated domain size and time formation.

In $\S 3$ we assume, more realistically, that the ratio of relevant time scales is small but not zero. The analytical problem is then considerably more difficult and results in an interesting and nontrivial singular perturbation problem which we discuss and analyse.

2. Preliminary analysis. The governing system of equations we consider for the model mechanism is (1) and (2). To simplify the analysis of this system, namely

$$
\begin{aligned}
& S_{t^{*}}^{*}=D \Delta^{*} S^{*}-k S^{*}, \\
& g_{t^{*}}^{*}=k_{1} S^{*}+\alpha g^{*}\left(g^{*}-k_{2}^{*}\right)\left(g_{c}-g^{*}\right)
\end{aligned}
$$

we nondimensionalize it by setting

$$
\begin{aligned}
& g^{*}=g_{c} g, \quad k_{2}^{*}=g_{c} k_{2}, \quad k t^{*}=t, \\
& \frac{k}{D} r^{* 2}=r^{2}, \quad k_{1} S^{*}=\alpha g_{c}^{3} S .
\end{aligned}
$$


In addition, we let $k / \alpha g_{c}^{2}=\varepsilon$, where $0<\varepsilon \ll 1$. This corresponds physically to the timescale of the biological switch being much smaller than that of the diffusing morphogen, as can be seen from the resulting equations,

$$
\begin{aligned}
& S_{t}=\Delta S-S, \\
& \varepsilon g_{t}=\left[S+g\left(g-k_{2}\right)(1-g)\right] .
\end{aligned}
$$

For simplicity, we take as initial conditions

$$
g(r, 0)=0, \quad S(r, 0)=\frac{S_{0}}{4 \pi t_{0}} e^{-r^{2} / 4 t_{0}-t_{0}} .
$$

The parameter $S_{0}$ measures the total amount of morphogen present and $t_{0}$ measures its initial spread; if $t_{0} \rightarrow 0$ then $S(r, 0) \rightarrow \delta(r)$, the delta function. (Thus, for $t_{0}$ small (7) gives a smooth but narrow initial distribution.) More general initial conditions can be treated but would not add greatly to the results.

In dimensionless form there are only four parameters: $S_{0}, t_{0}, k_{2}$ and $\varepsilon$. From the analysis we shall also see that in the limit $\varepsilon \rightarrow 0$ the basic solution behavior of (5) and (6) can be described by even fewer combinations of these dimensionless parameters. In fact, $\varepsilon$ is important only when the detailed structure is considered.

Note that for the initial condition (7) the solution of (5) is

$$
S(r, t)=\frac{S_{0}}{4 \pi\left(t+t_{0}\right)} \exp \left[\frac{-r^{2}}{4\left(t+t_{0}\right)}-\left(t+t_{0}\right)\right] .
$$

Thus, (6) reduces to

$$
\varepsilon g_{t}=S(r, t)+g\left(g-k_{2}\right)(1-g)
$$

where $S(r, t)$ is a known function and so the variable $r$ enters only as a parameter; we can therefore think of (9) as giving a separate ordinary differential equation for each different value of $r$.

Since the reaction time for the product $g$ is believed to be relatively short, we expect that most of the time this reaction will be near equilibrium. This quasi-steady state $g_{s}$ is obtained by setting the right-hand side of (9) to zero,

$$
S+g_{s}\left(g_{s}-k_{2}\right)\left(1-g_{s}\right)=0,
$$

or

$$
S+f\left(g_{s}\right)=0
$$

using more compact notation, and can be represented graphically as in Fig. 2.

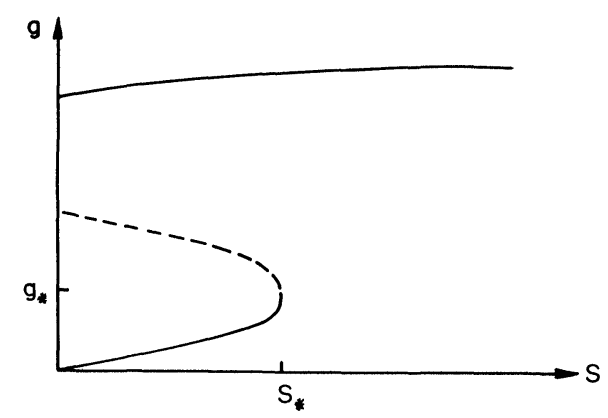

FIG. 2. The quasi-steady equilibria and their stability. (Solid lines are stable.) 
The stability of the quasi-steady states is easily determined; they are stable if

$$
f^{\prime}\left(g_{s}\right)<0 \quad\left(\text { or, } \frac{\partial S}{\partial g_{s}}>0\right) .
$$

The point $\left(S_{*}, g_{*}\right)$, where the steady states have a fold and where the stability changes, is found by solving $\partial S / \partial g_{s}=0$, which yields

$$
\begin{aligned}
& g_{*}=\frac{1+k_{2}}{3}-\frac{1}{3} \sqrt{1-k_{2}+k_{2}^{2}}, \\
& S_{*}=\frac{1}{9} k_{2}\left(1+k_{2}\right)-\frac{2}{9}\left(1-k_{2}+k_{2}^{2}\right) g_{*} .
\end{aligned}
$$

Given the zero initial conditions for $g$, there will first be a very rapid flow onto one of the quasi-steady states (timescale $O(\varepsilon)$ ). This is shown in Fig. 3.

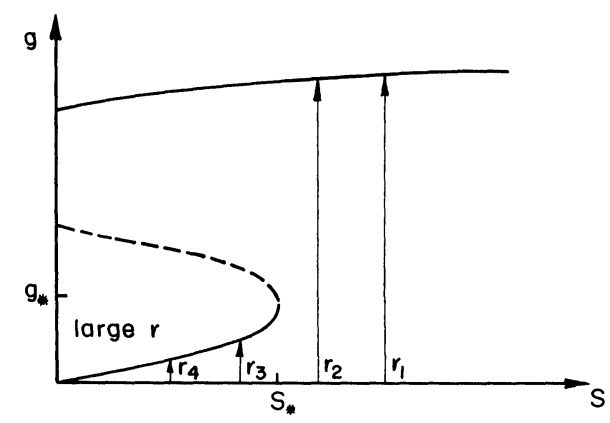

FIG. 3. Rapid flow from zero onto the quasi-steady equilibrium, for different values of $r\left(r_{1}<r_{2}<r_{3}<r_{4}\right)$.

Each value of $r$ has a different initial value of $S$ and thus generates a separate solution for $g$; large values of $S$ correspond to small values of $r$ and vice-versa.

For $S>S_{*}$ the rapid flow is onto the upper branch of the steady state curve, while for $S<S_{*}$ it is onto the lower branch. Since the upper branch represents activation of the biochemical switch, we see that $S_{*}$ is the critical value of $S$ for activation of this switch. Also important is the value of $r$ at which $S=S_{*}$, denoted $r_{c}(t)$. This is given by the solution of

$$
S_{*}=\frac{S_{0}}{4 \pi \tau} e^{-r_{c}^{2} / 4 \tau-\tau} \Rightarrow r_{c}^{2}(t)=-4 \tau \ln \left[\frac{S_{*}}{S_{0}} 4 \pi \tau e^{\tau}\right],
$$

where $\tau=t+t_{0}$. It will be shown shortly that in appropriate cases $r_{c}(t)$ gives the size of the activated zone.

After the rapid rise onto the quasi-steady state curve is completed, there is a slower flow along this curve (with timescale $O(1)$ ) since $S(r, t)$ is changing with time. Note, however, that even if $S$ decreases, once the solution gets onto the upper branch it stays there. Thus, once the biochemical switch is activated it stays on.

The slower flow along the steady-state curve is obviously governed by the sign of $\partial S / \partial t$. We know from the solution (8) of the diffusion equation that local maxima decrease with time, and therefore the largest values of $S$ will have $\partial S / \partial t<0$ (i.e. slow flow to the left). We also expect the smaller values of $S$ to have $\partial S / \partial t>0$ (slow flow to the right), at least initially (eventually the entire solution will decay with time).

The dividing line between these two behaviors is where $\partial S / \partial t=0$. From the solution (8) this occurs at a position

$$
r_{m}(t)=2 \sqrt{\tau+\tau^{2}}
$$


and value of $S$

$$
S\left(r_{m}(t), t\right)=\frac{S_{0}}{4 \pi \tau} e^{-1-2 \tau},
$$

where again $\tau=t+t_{0}$. Note that $r_{c}(t)$ has a maximum when $r_{c}(t)=r_{m}(t)$.

With this information a simple picture of the basic behavior can be given. As discussed previously, there is a quick flow onto the quasi-steady states and then slow flow along this curve. If $S\left(r_{m}(t), t\right)>S_{*}$ then the $r$ positions for which $S$ is near $S_{*}$ have $S$ increasing and the slow flow carries the solutions on the lower branch past the corner at $\left(S_{*}, g_{*}\right)$ and up onto the upper branch (see Fig. 4). Thus, the region in $r$ that has $g$ on the upper branch is growing, which is equivalent to saying $r_{c}(t)$ is increasing with time.

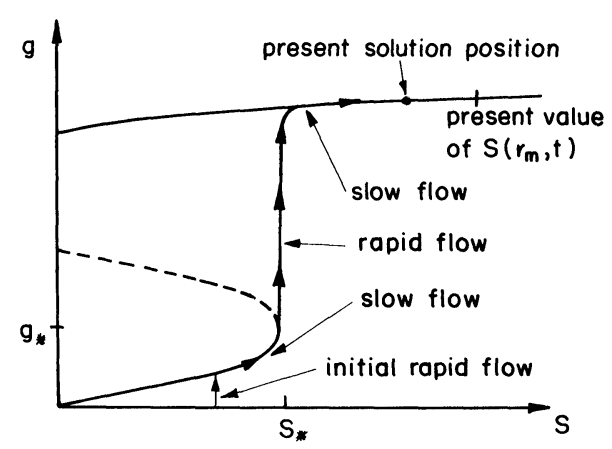

FIG. 4. Solution for a particular fixed $r$ value showing transition from lower to upper branch when $S\left(r_{m}, t\right)>S_{*}$.

As $t$ increases $r_{m}(t)$ increases and $S\left(r_{m}(t), t\right)$ decreases. The size of the region that has $g$ on the upper branch, which so far has been $r_{c}(t)$ to leading order, continues to increase. Finally $S\left(r_{m}(t), t\right)=S_{*}$ and $S(r, t)$ no longer increases (for fixed $r$ ) near $S_{*}$. Thus, solutions are no longer carried around the corner and the size of the region that has been switched on no longer continues to grow. For further times there is merely flow along the quasi-steady state curve, as shown in Fig. 5, and no more transitions occur.

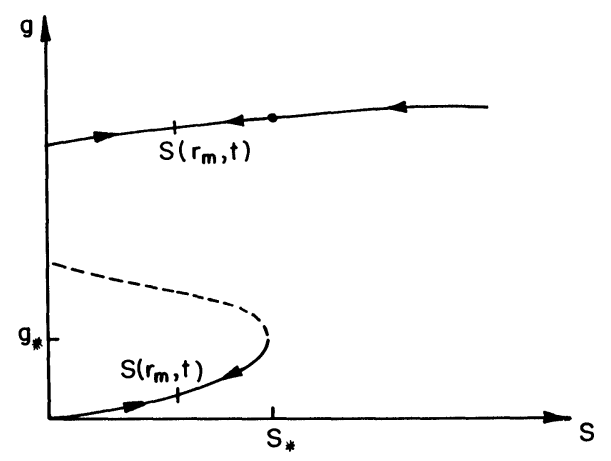

FIG. 5. Directions of slow flow along the quasi-steady curve when $S\left(r_{m}(t), t\right)<S_{*}$. Dot indicates possible present position of a solution that has made an earlier transition from the lower branch. 
To summarize, the size of the region that has been switched on is given by

(a) $r_{c}(t)$ if $S\left(r_{m}(t), t\right)>S_{*}$, or equivalently if $r_{m}(t)<r_{c}(t)$;

(b) $r_{*} \equiv r_{m}\left(t_{*}\right)=\max r_{c}(t)$ if $S\left(r_{m}(t), t\right)<S_{*}$ or if $r_{m}(t)>r_{c}(t)$ (where $t_{*}$ is defined by $\left.r_{m}\left(t_{*}\right)=r_{c}\left(t_{*}\right)\right)$.

Note that the above assumes that at $t=0 S\left(r_{m}(0), 0\right)>S_{*}$. by

If $S\left(r_{m}(0), 0\right)<S_{*}$ then the size of the region does not vary with time and is given

$$
r_{*}=r_{c}(0)
$$

if $r_{c}(0)>0$. Otherwise $r_{*}=0$ and no part of the region is switched on.

The reason for this latter part is that if $S\left(r_{m}(0), 0\right)<S_{*}$ then all values of $S$ with $S>S_{*}$ have $\partial S / \partial t<0$, so that the slow flow carries no solution around the corner, and the size of the region is determined by its initial size. If $r_{c}(0)=0$ or does not exist, then $S(r, 0) \leqq S_{*}$ for all $r$ and no $S$ is large enough to turn the switch on.

In terms of the dimensionless variables, we see that the size of the region switched on depends only upon the time $\tau=t+t_{0}$ and the ratio $S_{*} / S_{0}$. (Recall $S_{*}$ is a function of $k_{2}$.) Thus, we can plot $r(t)$ as a function of time for different values of $S_{*} / S_{0}$, as in Figs. 6 and 7. The second figure gives the results for when the size of the region is given by the initial condition; to use it merely find the correct values of $S_{*} / S_{0}$ and $t_{0}$ and read off the value of $r_{*}$.

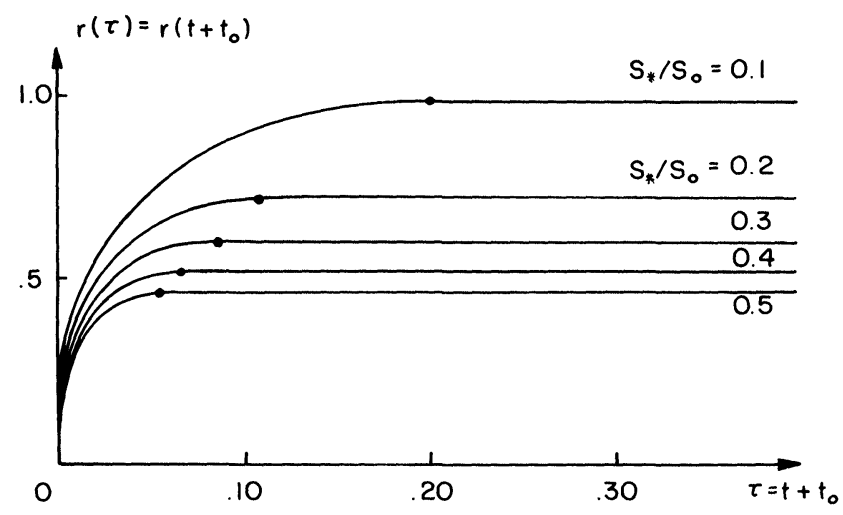

FIG. 6. Size of activated region if $S\left(r_{m}(0), 0\right)>S_{*}$.

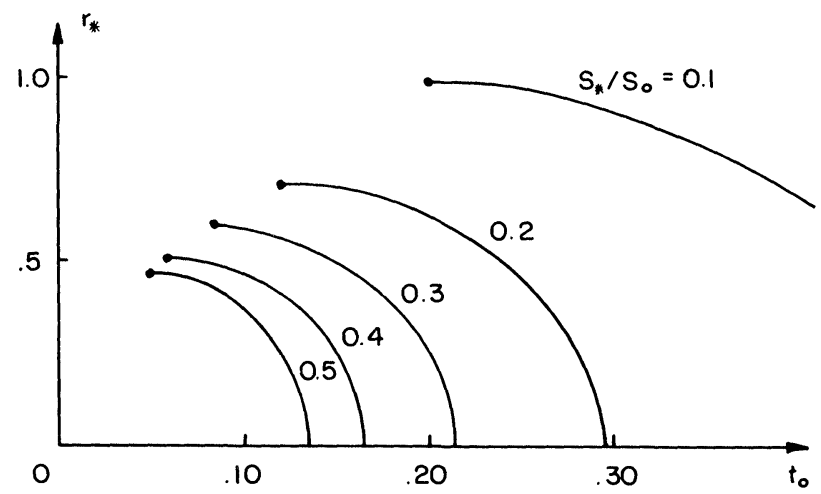

FIG. 7. Graph to give constant size of activated region if $S\left(r_{m}(0), 0\right)<S_{*}$. 
3. Detailed analysis. Given that $g(r, 0)=0$ there is initially a rapid flow onto the quasi-steady states, as we have seen. This can either be described by using boundarylayer theory (as done by Haberman [2]) or a multiple-scale expansion [3]. The latter, which we use, has the advantage of describing both the rapid flow and most of the slower flow along the steady-state curve.

First rescale the time

$$
t=\varepsilon \tilde{t}
$$

and (6) becomes

$$
\frac{d g}{d \tilde{t}}=S(r, t)+g\left(g-k_{2}\right)(1-g)=S(r, t)+f(g) .
$$

Now, $S$ varies on a timescale much slower than $\tilde{t}$, so expand

$$
g \sim g_{0}(\tilde{t}, t)+\varepsilon g_{1}(\tilde{t}, t)+\cdots
$$

and substitute into (22). (If the constant $t_{0}=0$ then $S(r, t)$ has a singularity near $t=0$ but this can be remedied by a further scaling near $t=0$.) To $O(1)$ in $\varepsilon$,

$$
\frac{\partial g_{0}}{\partial \tilde{t}}=S(r, t)+f\left(g_{0}\right)
$$

which when combined with the initial condition $g_{0}(0,0)=0$ can be integrated to yield

$$
g_{0}=g_{0}(\tilde{t}, t)
$$

(the arbitrary function of $t$ arising in the integration is unimportant and is taken to be zero). Note that

$$
\lim _{\tilde{t} \rightarrow \infty} g_{0}(\tilde{t}, t)=g_{s}(t)
$$

is the quasi-steady state solution, and that the slow variation is automatically determined with this procedure (it comes in through the parametric variation of $S$ with $t$ ).

To $O(\varepsilon), g_{1}$ is given by

$$
\frac{\partial g_{1}}{\partial \tilde{t}}-f^{\prime}\left(g_{0}\right) g_{1}=-\frac{\partial g_{0}}{\partial t}
$$

which has the integrating factor $\left(\partial g_{0} / \partial \tilde{t}\right)^{-1}$ and $g_{1}(0,0)=0$, yielding

$$
g_{1}=-g_{0_{\tilde{t}}} \int_{0}^{\tilde{t}} \frac{g_{0_{t}}(\zeta, t)}{g_{0_{\tilde{i}}}(\zeta, t)} d \zeta .
$$

Note now that

$$
\lim _{\tilde{t} \rightarrow \infty} g_{1}(\tilde{t}, t)=-\frac{1}{f^{\prime 2}\left(g_{s}\right)} \frac{\partial S}{\partial t}
$$

and so if $f^{\prime}\left(g_{s}\right) \rightarrow 0$ then $g_{1}$ becomes unbounded as $\tilde{t} \rightarrow \infty$, and the expansion breaks down.

This breakdown occurs as the fold $\left(S_{*}, g_{*}\right)$ is approached, as was anticipated earlier. The way the solution breaks down can be examined to determine the new scaling (see [2]), but it is perhaps easier to determine this directly from the equation.

The scaling of the solution near $\left(S_{*}, g_{*}\right)$ gives rise to the first type of transition layer, the first possible way of having a transfer from the lower to the upper branch 
of the steady-state curve. (This scaling also provides the solutions necessary to complete the rapid initial flow onto the quasi-steady states, the part with $S$ near $S_{*}$.) Suppose $S(r, t)=S_{*}$ when $t=t_{c}(r)$, and suppose that $t-t_{c}$ is small (we will from now on suppress all $r$ dependence unless it adds to the discussion). In particular, let

$$
t=t_{c}+\delta(\varepsilon) \tilde{t}
$$

with $0<\delta \ll 1$, so that

$$
S(t)=S_{*}+S^{\prime}\left(t_{c}\right) \delta(\varepsilon) \tilde{t}+\cdots,
$$

where $S^{\prime}$ means $\partial S / \partial t$. Also assume $g$ is near $g_{*}$ and let

$$
g=g_{*}+\nu(\varepsilon) \tilde{g}
$$

where $0<\nu \ll 1$.

With these assumptions equation (6) for $g$ becomes

$$
\varepsilon \frac{\nu}{\delta} \frac{d \tilde{g}}{d \tilde{t}}=\frac{1}{2} f^{\prime \prime}\left(g_{*}\right) \nu^{2} \tilde{g}^{2}+\delta S^{\prime}\left(t_{c}\right) \tilde{t}+\cdots
$$

since $f^{\prime}\left(g_{*}\right)=0$ (note $f^{\prime \prime}\left(g_{*}\right)>0$ ). For all of these terms to balance, we must have

$$
\varepsilon \frac{\nu}{\delta}=\nu^{2}=\delta
$$

which results in

$$
\nu=\varepsilon^{1 / 3}, \quad \delta=\varepsilon^{2 / 3},
$$

and (32) becomes

$$
\frac{d \tilde{g}}{d \tilde{t}}=\frac{1}{2} f^{\prime \prime}\left(g_{*}\right) \tilde{g}^{2}+S^{\prime}\left(t_{c}\right) \tilde{t}
$$

to leading order.

This can be further simplified by setting

$$
\tilde{t}=\left(\frac{1}{2} f^{\prime \prime}\left(g_{*}\right) S^{\prime}\left(t_{c}\right)\right)^{-1 / 3} z, \quad \tilde{g}(\tilde{t})=\left(\frac{S^{\prime}\left(t_{c}\right)}{\frac{1}{4} f^{\prime \prime 2}\left(g_{*}\right)}\right)^{1 / 3} w(z),
$$

and (33) becomes

$$
\frac{d w}{d z}=w^{2}+z
$$

If we now let

$$
w=-\frac{1}{y} \frac{d y}{d z}
$$

the last equation becomes the Airy equation [1]

$$
\frac{d^{2} y}{d z^{2}}=-z y
$$

Thus, the solution $w$ of (35) is

$$
w=\frac{\operatorname{Ai}^{\prime}(-z)+c \mathrm{Bi}^{\prime}(-z)}{\operatorname{Ai}(-z)+c \operatorname{Bi}(-z)} .
$$


The constant $c$ is determined by matching with the solution prior to entering the region near $\left(S_{*}, g_{*}\right)$, either with the initial layer solution or with the quasi-steady state solution. If we match with the latter solution, then we find $c=0$ by using the asymptotics of the Airy functions. This corresponds to a solution following the quasi-steady state solution and being pushed past the corner. If we wish to match with the initial layer, then $c$ must be chosen so that the denominator

$$
\mathrm{Ai}(-z)+c \mathrm{Bi}(-z)
$$

has a zero in the appropriate place. The solution near this pole can then be used to match onto the initial boundary layer. (The only time it will be necessary to use nonzero values of $c$ in the matching is near $t=0$ and $r=r_{c}(0)$, i.e., for matching to those initial layers with $S(r, 0)$ near $S_{*}$. In most cases when the transition layer is needed, however, the solution will already have had sufficient time to flow onto the lower quasi-steady curve so that $c=0$ is appropriate.)

Note that $A(-z)$ and $B(-z)$ are both oscillatory for $z>0$; this means that no matter what the constant $c$ there is a pole for $z>0$. For example, if $c=0$ then

$$
w=\frac{\operatorname{Ai}^{\prime}(-z)}{\operatorname{Ai}(-z)} \sim \frac{1}{\alpha-z} \quad \text { as } z \rightarrow \alpha^{-},
$$

where $\alpha$ is the first positive zero of $\mathrm{Ai}(-z)$.

This shows that all solutions round the corner and match onto another boundary layer solution, which takes them onto the upper branch. The details of the matching of the solutions near the poles with the boundary layers and the flow onto the upper branch do not add much to the discussion, and will be omitted (see [2] for the details).

The above analysis for the transition layers breaks down for values of $\boldsymbol{r}$ for which $S^{\prime}\left(t_{c}\right) \rightarrow 0$. This is when the maximum of $S$ occurs near $S_{*}$. To analyze this case, which yields a second type of transition layer, we assume that the maximum occurs at $t_{m}$, and rescale using

$$
t=t_{m}+\delta(\varepsilon) \tilde{t}
$$

In addition, we assume

$$
S\left(t_{m}\right)=S_{*}+\eta(\varepsilon) S
$$

and again expand using

$$
g=g_{*}+\nu(\varepsilon) g \text {. }
$$

(Note that $t_{m}(r)$ is determined implicitly by $(\partial S / \partial t)\left(r, t_{m}\right)=0$. Since the definition of $t_{c}(r)$ was $S\left(r, t_{c}\right)=S_{*}$ and we are assuming $S\left(r, t_{m}\right)=S_{*}+o(1)$, this means that $t_{m}$ and $t_{c}$ will be close to each other in this case but not identical.)

In this case the resulting equation for $\tilde{g}$ equivalent to (32) is

$$
\varepsilon \frac{\nu}{\delta} \frac{d \tilde{g}}{d \tilde{t}}=\eta \tilde{S}+\frac{1}{2} S^{\prime \prime}\left(t_{m}\right) \delta^{2} \tilde{t}^{2}+\frac{1}{2} f^{\prime \prime}\left(g_{*}\right) \nu^{2} \tilde{g}^{2}+\cdots
$$

and balancing terms requires

$$
\frac{\varepsilon \nu}{\delta}=\eta=\delta^{2}=\nu^{2}
$$

which gives

$$
\nu=\delta=\varepsilon^{1 / 2}, \quad \eta=\varepsilon
$$


Equation (37) can again be further simplified, in this case by defining

$$
\begin{aligned}
& \tilde{t}=\left(\frac{-1}{f^{\prime \prime}\left(g_{*}\right) S^{\prime \prime}\left(t_{m}\right)}\right)^{1 / 4} z, \\
& \tilde{g}(\tilde{t})=2\left(\frac{-S^{\prime \prime}\left(t_{m}\right)}{f^{\prime \prime}\left(g_{*}\right)}\right)^{1 / 4} w(z), \\
& \tilde{S}=2\left(\frac{-S^{\prime \prime}\left(t_{m}\right)}{f^{\prime \prime}\left(g_{m}\right)}\right)^{1 / 2}\left[\kappa+\frac{1}{2}\right],
\end{aligned}
$$

which gives

$$
\frac{d w}{d z}=w^{2}+\left(\kappa+\frac{1}{2}\right)-\frac{1}{4} z^{2}
$$

If we now let

$$
w=-\frac{1}{y} \frac{d y}{d z}
$$

the result is

$$
\frac{d^{2} y}{d z^{2}}+\left[\kappa+\frac{1}{2}-\frac{1}{4} z^{2}\right] y=0,
$$

the solutions of which are parabolic cylinder functions [1].

As for the previous case the constants in the solution are determined by matching with the solution prior to entering the region near $\left(S_{*}, g_{*}\right)$; now, however, we expect the generic case of the matching to be with the quasi-steady state solution. This is because in general the transients (i.e. boundary layer solutions) will have died out by the time $S\left(r_{m}(t), t\right)$ decreases to $S_{*}$. The only time we will need to match the boundary layer solution to this second type of transition layer is near $t=0$ and when $S\left(r_{m}(0), 0\right) \cong$ $S_{*}$, which is unlikely.

To match with the quasi-steady state solution we require

$$
w \sim \frac{z}{2} \text { as } z \rightarrow-\infty
$$

which means that $y$ from (39) must decay exponentially (like $e^{-z^{2} / 4}$ ) as $z \rightarrow-\infty$. This fixes the solution as

$$
y=c D_{\kappa}(-z),
$$

which gives, as the appropriate solution of (38),

$$
w=\frac{D_{\kappa}^{\prime}(-z)}{D_{\kappa}(-z)} \text {. }
$$

(Then $y \sim(-z)^{\kappa} e^{-z^{2} / 4}$ and $w \sim z / 2$ as $z \rightarrow-\infty$.)

To find out what happens to the solution as $z$ increases, we need to know something about $D_{\kappa}(-z)$. If $\kappa<0$ then there are no real zeros of $D_{\kappa}(-z)$. Thus, $w$ in $(40)$ remains bounded for all finite $z$, and what happens is determined by the asymptotic behavior of $D_{\kappa}(-z)$ as $z \rightarrow+\infty$, which is

$$
D_{\kappa}(-z) \sim-\frac{(2 \pi)^{1 / 2}}{\Gamma(-\kappa)} z^{-\kappa-1} e^{z^{2} / 4} \quad \text { as } z \rightarrow+\infty .
$$


Thus, from (40),

$$
w \sim-\frac{z}{2} \text { as } z \rightarrow+\infty
$$

which means that the solution again approaches the lower, stable branch of the quasi-steady state solution. This makes sense, since when $\kappa$ is large and negative the solution stops far short of the fold before it turns back.

If $\kappa>0$, on the other hand, then $D_{\kappa}(-z)$ does have zeros, and therefore $w$ will have a pole at the first zero,

$$
w \sim \frac{1}{\alpha-z}+\cdots \quad \text { as } z \rightarrow \alpha^{-},
$$

$\alpha$ being the first zero of $D_{\kappa}(-z)$. This solution will match onto a boundary layer which will then take the solution from near $\left(S_{*}, g_{*}\right)$ onto the upper stable branch. Thus, if $\kappa>0$ there is enough push to round the corner and make the transition onto the upper branch.

This is not uniform in $\kappa$, however, because as $\kappa \rightarrow 0^{+}$the zero $\alpha$ moves out to $+\infty$, so that one must wait longer and longer (in $z$ ) before being able to match onto the boundary layer. Finally, when $\kappa=0$ something interesting happens. In this case

$$
D_{0}(-z)=e^{-z^{2} / 4}
$$

exactly, and therefore

$$
w=\frac{z}{2}
$$

exactly as well. Thus, this solution neither has a pole to match onto a new boundary layer nor does it return to the lower branch.

Although this transition layer is only approximate, it suggests the existence (probably justified by a higher order analysis) of a solution which starts on the lower stable branch of the quasi-steady state solution, just manages to round the corner and then approaches the unstable branch. This solution (given to leading order by $\kappa=0$ ) is the dividing line between the solutions which eventually approach the upper stable branch and those which return to the lower one.

At this point it should be remembered that $\kappa$ is a function of $r$. (In fact, for a fixed value of $r, \kappa$ is basically a shifted and scaled measure of the difference between the maximum value of $S$ at this position and $S_{*}$.) Thus, there is a particular value of $r$ that gives $\kappa=0$; for slightly smaller values of $r, \kappa$ is positive and for slightly larger values, $\kappa$ is negative. At some fixed time after the $\kappa=0$ solution has rounded the corner there will be some solutions with $\kappa>0$ that have reached the upper branch, and some with $\kappa$ only very slightly positive that have just recently left the neighborhood of the unstable branch. There is also similar behavior for the solutions with $\kappa<0$ that eventually return to the lower branch.

Considered as a function of $r$, therefore, at this fixed time there will be a continuous but steep transition between the lower and upper branches, as in Fig. 8. This transition represents the boundary between the activated and unactivated regions of the biochemical switch, and will become increasingly sharper as time progresses.

Note that to $O(\varepsilon)$ the critical value of $S$ for activation of the biological switch is

$$
S_{*}+\left(-\frac{S^{\prime \prime}\left(t_{m}\right)}{f^{\prime \prime}\left(g_{m}\right)}\right)^{1 / 2} \varepsilon+\cdots
$$




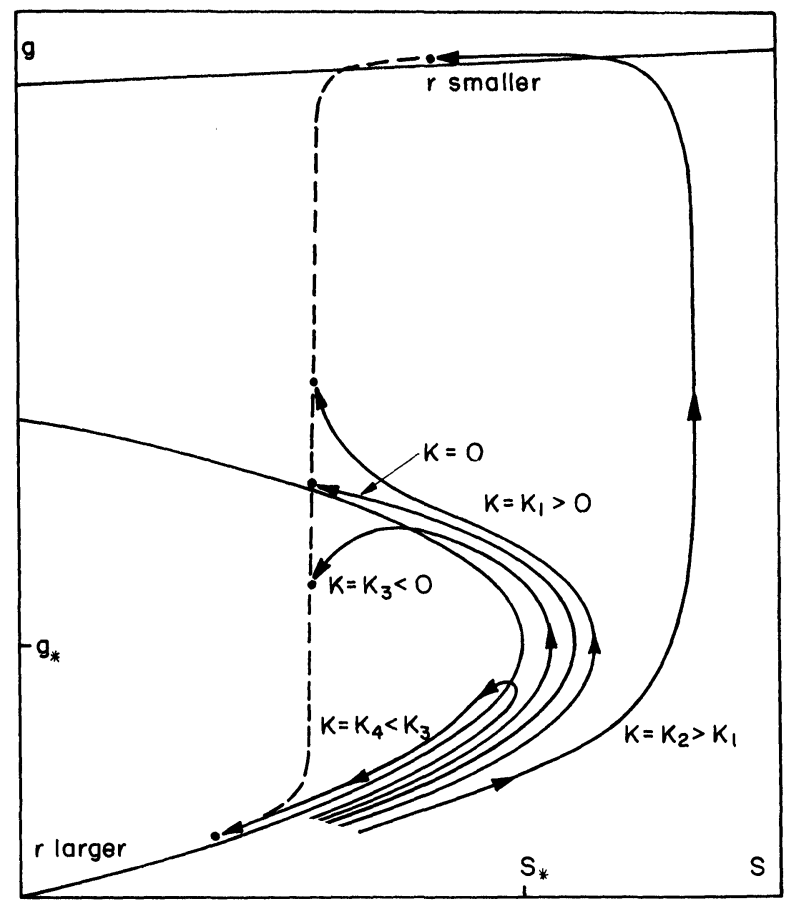

FIG. 8. Behavior of second type of transition layer. Solid lines give time history of solutions; dotted lines give present $r$ dependence.

(i.e. $\kappa=0$ ), which is slightly larger than $S_{*}$. Thus, the final size of the activated region will be slightly smaller than that predicted in $\S 2$.

4. Biological relevance of the analysis. Although there is now a substantial body of evidence for a morphogen controlled pattern formation mechanism for butterfly and moth wings, the identification of an actual morphogen has proved elusive. Using the $\varepsilon=0$ analysis in $\S 2$ for the radius of the eyespot, a best fit comparison with Nijhout's [6] experiments at two different temperatures determined approximate values for the various parameters. The effective in vivo tissue diffusion coefficient obtained is $O\left(10^{-9}-10^{-11} \mathrm{~cm}^{2} \mathrm{sec}^{-1}\right)$, which although small is now considered to be of the right order of magnitude expected for any morphogen in a real situation where diffusion across cells involves passage through gap junctions. The diffusion time scale associated with such a diffusion coefficient is $O\left(L^{2} \times 10^{9}-10^{11} \mathrm{sec}\right)$, where $L$ is a typical length (in $\mathrm{cm}$ ) associated with the pattern. Although large, it is not unduly so since the eyespots develop over a period of about 4-8 days. Since the pattern develops progressively, it is essential that the reaction time associated with the product $g$ in our model is not as long. So it is reasonable to take the ratio of time scales, proportional to $\varepsilon$, to be small but not zero. If $\varepsilon$ was $O(1)$ the transition region would be very fuzzy-this is not the case with lepidopteran wing patterns such as we are modelling here. In fact they are quite sharp.

The analysis given in this paper determines the size of the small but finite transitions between the different regions which make up the eyespot at the various stages of its development. (The width is $O\left(\varepsilon^{2 / 3}\right)$ during initial formation, shrinks to $O(\varepsilon)$ as the pattern nears its final size and shape, and then decreases to the order of a cell diameter as the steady state is approached.) As has been the case in other models, for example 
the wave model for Belousov-Zhabotinskii waves (Murray [8]), an estimate of the transition width in terms of the parameters can prove useful in estimating them when accurate comparison is made with the dynamical experimental results.

The effect of temperature on the size of wing spots has been considered by Nijhout [6] and the best fit mentioned above took this into account. The nondimensionalization shows that although temperature affects both the rate constants and the diffusion coefficient these parameters appear in a composite way. If temperature influences on these parameters could be assessed individually, or even qualitatively, then our $O(\varepsilon)$ analysis would provide a more useful comparison with the transition layer's between one melanin and another. This would help in determining whether or not our model mechanism is a reasonable one or at least what qualitative properties the pattern formation mechanism must exhibit.

Some of the results obtained by Nijhout [5] involved transplanting incipient eyespot centers. We believe that if the area of the transplant is varied and the center production of morphogen is of comparatively short duration, then the size of the transplant should be of some importance. If the morphogen is produced continuously by a few cells over a longer period of time, the size of the transplanted eyespot should depend only weakly on the size of the transplanted region, and more on the time at which it is moved.

\section{REFERENCES}

[1] M. Abramowitz and I. A. Stegun, Handbook of Mathematical Functions, Dover, New York, 1964.

[2] R. HABERMAN, Slowly varying jump and transition phenomena associated with algebraic bifurcation problems, this Journal, 37 (1979), pp. 69-106.

[3] J. Kevorkian ANd J. D. Cole, Perturbation Methods in Applied Mathematics, Springer-Verlag, New York, 1981.

[4] J. D. MURRAY, On pattern formation mechanisms for lepidopteran wing patterns and mammalian coat markings, Phil. Trans. Roy. Soc. London B, 295 (1981), pp. 473-496.

[5] H. F. NiJHout, Wing pattern formation in Lepidoptera: a model, J. Exp. Zool., 206 (1978), pp. 119-136.

[6] _-, Pattern formation in lepidopteran wings: determination of an eyespot, Devel. Biol., 80 (1980a), pp. 267-274.

[7] - Ontogeny of the color pattern on the wings of Precis coenia (Lepidoptera: Nymphalidae), Devel. Biol., 80 (1980b), pp. 275-288.

[8] J. D. MURRAY, On travelling wave solutions in a model for the Belousov-Zhabotinskii reaction, J. Theor. Biol., 56 (1976), pp. 329-353. 